\title{
The NA62 GigaTracker
}

\section{Mathieu Perrin-Terrin* ${ }^{* \dagger}$}

CERN, Geneva, Switzerland

E-mail: mathieu.perrin-terrinecern.ch

The GigaTracker is an hybrid silicon pixel detector built for the NA62 experiment aiming at measuring the branching fraction of the ultra-rare kaon decay $K^{+} \rightarrow \pi^{+} v \bar{v}$ at the CERN SPS. The detector has to track particles in a beam with a flux reaching $1.3 \mathrm{MHz} / \mathrm{mm}^{2}$ and provide single-hit timing with 200ps RMS resolution for a total material budget of less than $1.5 X_{0}$. The tracker comprises three $60.8 \mathrm{~mm} \times 27 \mathrm{~mm}$ stations installed in vacuum $\left(\sim 10^{-6} \mathrm{mbar}\right)$ and cooled with liquid $\mathrm{C}_{6} \mathrm{~F}_{14}$ circulating through micro-channels etched inside few hundred of microns thick silicon plates. Each station is composed of a $200 \mu \mathrm{m}$ thick silicon sensor readout by $2 \times 5$ custom $100 \mu \mathrm{m}$ thick ASIC, called TDCPix. Each chip contains $40 \times 45$ asynchronous pixels, each $300 \mu \mathrm{m} \times 300 \mu \mathrm{m}$ and is instrumented with $100 \mathrm{ps}$ bin time-to-digital converters. In order to cope with the high rate, the TDCPix is equipped with four $3.2 \mathrm{~Gb} / \mathrm{s}$ serialisers sending out the data. We will describe the detector and the results from the 2014 NA62 run.

24th International Workshop on Vertex Detector -VERTEX2015-

\section{1-5 June 2015}

Santa Fe, New Mexico, USA

\footnotetext{
* Speaker.

${ }^{\dagger}$ On behalf of the GTK working group.
} 


\section{Introduction}

The GigaTracker is an hybrid silicon pixel detector built for the NA62 experiment at the CERN SPS. The NA62 experiment aims at measuring the branching fraction of the ultra-rare kaon decay $K^{+} \rightarrow \pi^{+} v \bar{v}$, a golden channel to look for physics beyond the standard model (SM) and to measure the CKM matrix element $\mathrm{V}_{t d}$ [2].

With a single charged particle in the final state and a decay branching fraction as small as $(7.81 \pm 0.80) \times 10^{-11}$ in SM [1], this measurement represents an experimental challenge. The resolution on the decay squared missing mass, $\left|p_{K}^{(4)}-p_{\pi}^{(4)}\right|^{2}$, is one of the key parameters of the experimental strategy and requires an accurate measurement of the initial state momentum. Another important parameter is the beam rate, which ought to be as high as possible in order to collect enough decays. Finally, the experiment beam being not bunched, it is necessary that all detectors provide timing information in order to link unambiguously initial and final states. The GigaTracker was designed to match these requirements which are described more precisely in Table 1.

Table 1: Specifications for the GigaTracker.

\begin{tabular}{|cc|}
\hline Beam Rate & $\begin{array}{c}800 \mathrm{MHz}-1 \mathrm{GHz} \\
1.3 \mathrm{MHz} / \mathrm{mm}^{2}\end{array}$ \\
\hline Radiation & $10^{14} 1 \mathrm{MeV}$ eq. $\mathrm{n} / \mathrm{cm}^{2} / \mathrm{y}$ \\
\hline Efficiency & $99 \%$ \\
\hline Momentum Resolution & $0.2 \%$ \\
\hline Angular Resolution & $16 \mu \mathrm{rad}$ \\
\hline Hit Time Resolution & $200 \mathrm{ps} \mathrm{RMS}$ \\
\hline Material Budget & $3 \times 0.5 \% X_{0}$ \\
\hline Detector Size & $60.8 \mathrm{~mm} \times 27 \mathrm{~mm}$ \\
\hline
\end{tabular}

The detector design opted for three stations of hybrid silicon pixels for which an applicationspecific integrated circuit (ASIC) -the TDCPix- has been developed using $130 \mathrm{~nm}$ technology and produced by IBM. The three stations are installed around two achromats, as show in Figure 1. By measuring how much a particle is shifted by the achromats, one measures the particle momentum.

The TDCPix ASIC was specifically developed for the GigaTracker needs and incorporates all the electronics needed to perform accurate hit arrival time measurements. The chip and the time measurement principle are described in Section 2. The integration of the chips (bump-bonded onto a sensor) are described in Section 3. Their integration covers mechanical, electrical and, thermal aspects and it is worth mentioning that the GigaTracker is the first detector to be cooled with micro-channel devices. The performance obtained during tests and in real experimental running conditions is described in Section 4. Conclusions and prospects will be discussed in Section 5.

\section{The TDCPix ASIC Assembly}

The large GigaTracker hybrid silicon pixel matrix is made by bump-bonding two rows of five TDCPix chips onto a $200 \mu \mathrm{m}$ thick silicon sensor of $60.8 \mathrm{~mm} \times 27 \mathrm{~mm}$ as shown in Figure 2. Both 


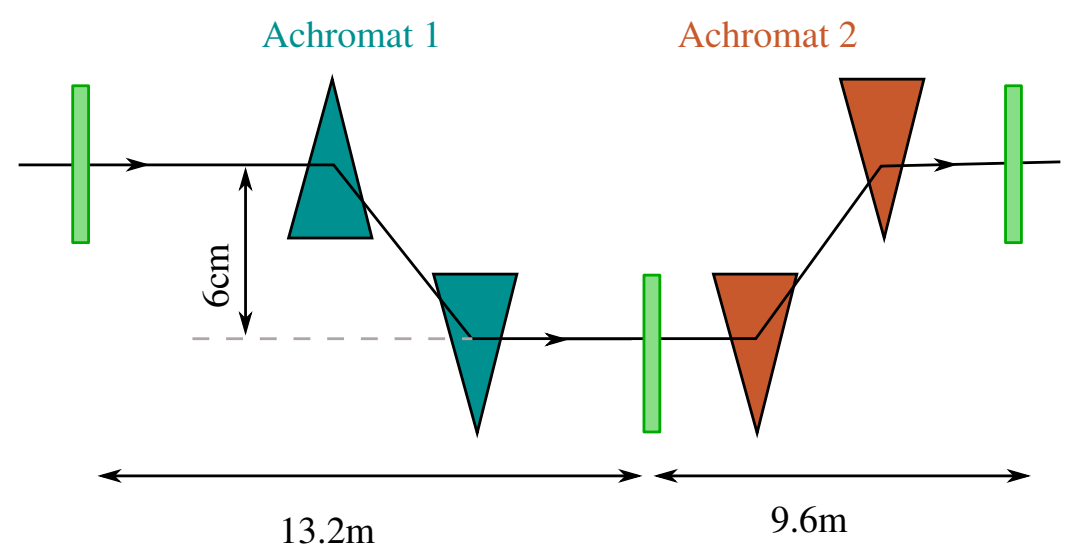

Figure 1: Layout of the GigaTracker. Three stations (green) are inserted around two achromats. The first one (blue) displaces the beam by $6 \mathrm{~cm}$ in average in the vertical plane. The second one (orange) brings the beam back on its original trajectory. The precise measurement of the vertical shift allows to measure the particle momentum.

p-in-n and n-in-p sensors can be used as the chip accepts both bias polarities. In order to match the material budget specifications, the ASICs have to be thinned to $100 \mu \mathrm{m}$. This thickness and the large size of sensor make the bump-bonding a very delicate step of the detector construction. This step was realised by the IZM company.

The chip architecture has been designed to keep the analog logic of the 1800 pixel array separated from the digital and time-to-digital conversion (TDC) logic sitting therefore outside of the pixel array, in the region called end-of-column as shown in Figure 2. The pixel matrix is organized in 40 columns of 45 pixels each. The pixel size is $300 \mu \mathrm{m} \times 300 \mu \mathrm{m}$. Even though the junction is fully depleted with a bias of around 50-60 V, the sensor is operated at a larger bias voltage up to $450 \mathrm{~V}$, such that the charges created by a particle crossing the sensor drift through the junction in a time interval smaller than $10 \mathrm{~ns}$. For minimum-ionizing particles the charge deposition follows a Landau distribution and the most probable value for a $200 \mathrm{mu}$ thick detector is $2.4 \mathrm{fC}$. Each pixel electrode is connected to a pre-amplifier with a gain of $70 \mathrm{mV} / \mathrm{fC}$. The output of the pre-amplifier is connected to a discriminator with a tunable threshold. To be immune against bit flits caused by single event upsets, three sets of register are voting each pixel threshold value. The discriminated signals are then transmitted to the end-of-column.

The preamplifier is designed to give a peaking time of $5 \mathrm{~ns}$ regardless of the signal amplitude. Thus higher signal pulses will cross the discriminator threshold earlier than lower pulses as shown in Figure 3. The signal time-walk can be as big as several nano-seconds and therefore must be corrected in order to reach the 200ps hit arrival time resolution needed by the experiment. This correction is performed by measuring both the leading and trailing edges of the pulse. The difference of the two, called time-over-threshold, is a proxy to the signal amplitude and can therefore be used to estimate the time-walk ${ }^{1}$. The time-walk as a function of the time-over-threshold is determined statistically and then applied to individual hits.

\footnotetext{
${ }^{1}$ This holds in the linearity regime of the preamplifier, whilst for signals saturating the preamplifier the corrections are however negligible.
} 
The measurement of the leading and trailing time is performed by TDCs sitting in the TDCPix end-of-columns. Overall the TDCPix contains 360 pairs of TDC with 100ps bins. Five pixels are therefore sharing a TDC pair. These pixels are distributed within a column such that their spacing is maximized. Hence a particle cannot deposit charge in two pixels of the same group.

In order to cope with the high hit rate each 10 column-group, or quarter-chip, is equipped with a $3.2 \mathrm{~Gb} / \mathrm{s}$ serialisers sending the hit data out through a printed circuit board (PCB) described in the next section.

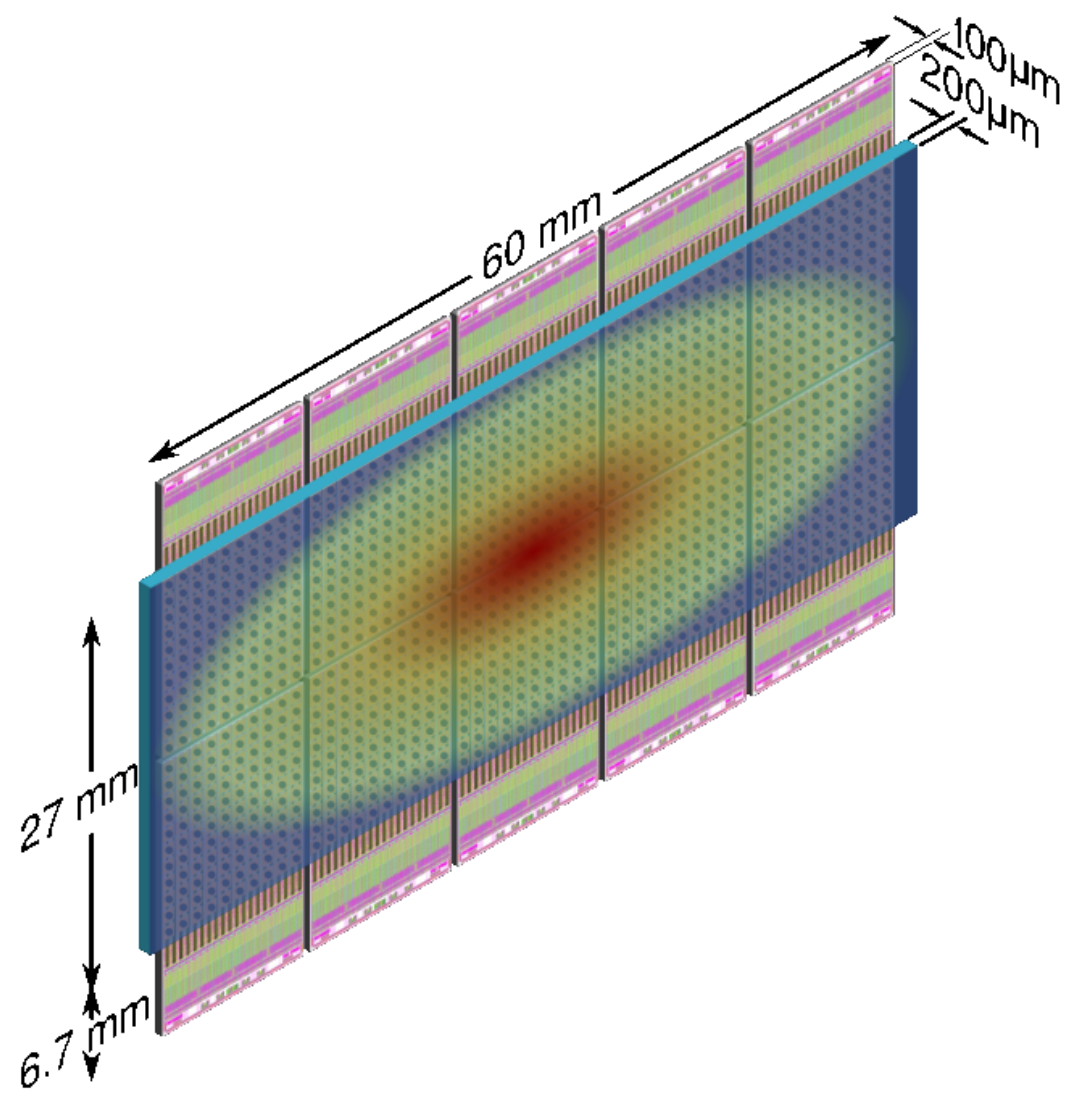

Figure 2: The TDCPix assembly is composed of a $60.8 \mathrm{~mm} \times 27 \mathrm{~mm}$ sensor bump-bonded onto $5 \times 2$ TDCPix chips. The chip digital and time-to-digital converters logic is located in the $6.7 \mathrm{~mm}$ extending outside the sensor.

\section{Detector Integration}

The detector integration covers aspects ranging from mechanics, electronics and cooling. It is worth reminding that the detector is sitting directly in the beam and in vacuum. These conditions that have of courses a strong implications on the integration design.

\subsection{Mechanical Integration}

The GigaTracker mechanical integration is illustrated on Figure 4. The detector is taped onto a few hundred of microns thick micro-channel cooling plate that can be considered in this subsection 


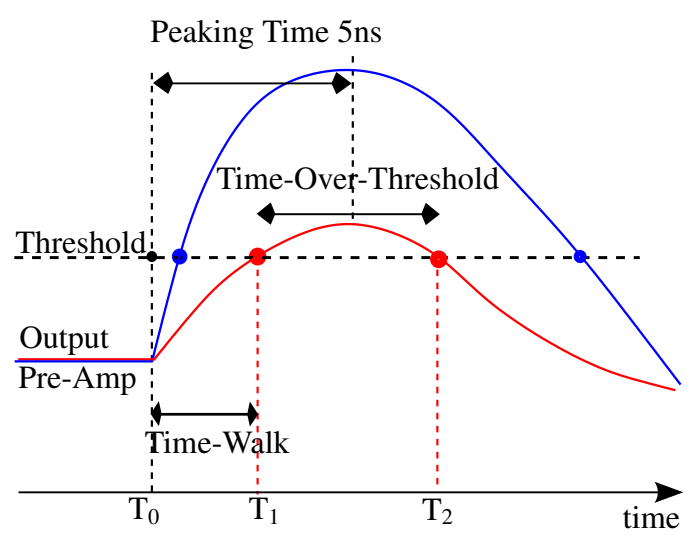

(a)

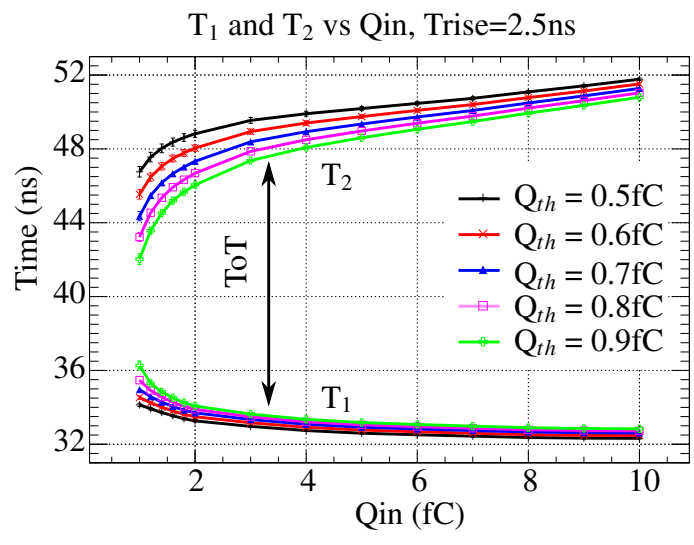

(b)

Figure 3: (a) Pulses with large amplitude have shorter time-walk and larger time-over-threshold than smaller pulses (courtesy M. Noy). (b) Leading $\left(\mathrm{T}_{1}\right)$ and trailing $\left(\mathrm{T}_{2}\right)$ time as a function of charge deposition from [3].

simply as a mechanical support. This plate is then clamped onto the sides of a pool countersunk into the PCB. The clamping is made at three points to not over-constrain the silicon plate. The PCB is glued into a frame and a flange. The whole assembly is slided into the vacuum vessel and the flange closes it hermetically making also the feed-through for the cooling liquid.

\subsection{Electrical Integration}

The PCB is the key element for the electrical integration of the detector. It has to ship the data sent by the forty $3.2 \mathrm{~Gb} / \mathrm{s}$ serial link of the detector outside of the vacuum vessel. Therefore the strips onto which these data signal are transiting can be as long as $30 \mathrm{~cm}$. In addition the PCB has to connect to the configuration pads of the chips. As a result, not less than 14 internal layers are needed to route all the signals.

At the detector side the connection to the TDCPix is made through a dense set of wire-bonds with adjacent pads as close as $7.3 \mu \mathrm{m}$ as shown in Figure 5. The PCB playing also the role of mechanical support, its planarity must also be kept below few ten of microns around the countersink.

Outside the vessel, the PCB is equipped with optical links connected through $300 \mathrm{~m}$ long fibres to data acquisition boards located outside the experimental area. The clock, configuration, resets and, data are transmitted on those links. Each TDCPix is controlled and read-out by one DAQ board. These boards are also connected to the experiment trigger system and reply to trigger requests by retrieving in their $1 \mathrm{~ms}$ buffers all hits within $75 \mathrm{~ns}$ around the trigger time stamp.

\subsection{Thermal Integration}

The TDCPix dissipates around 3.5W, mostly in the end-of-column region where all the digital logic stands. Being the detector in vacuum, the heat has to be removed by a dedicated system. In addition, the damage caused by the beam radiation can be reduced by operating the sensor at low temperature. The solution opted for the cooling system employs micro-channel cooling devices, a technology never used before in particle physics. The main advantage of this technology is the minute amount of material that is required to cool the detectors. A few hundred of microns thick 
]

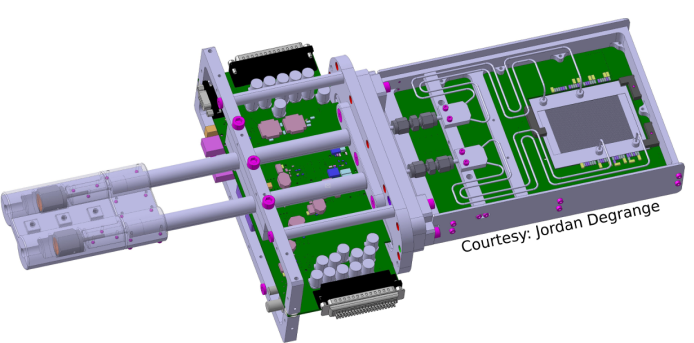

(a)

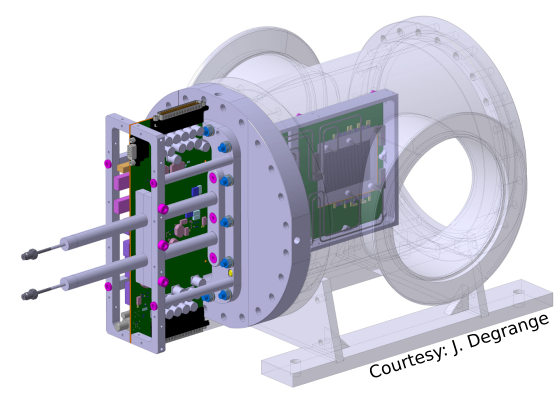

(c)

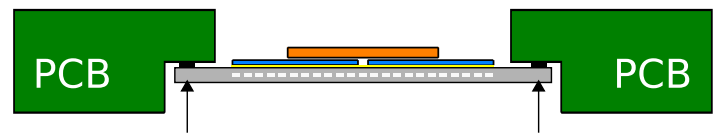

(b)

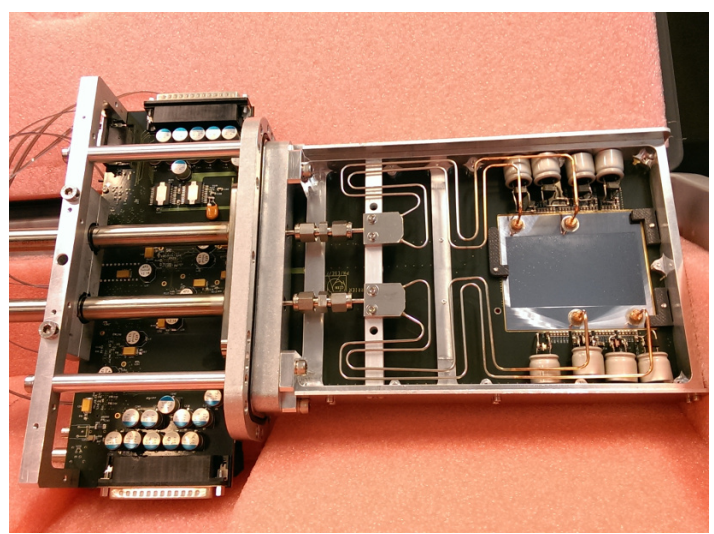

(d)

Figure 4: (a) Schematics of a GigaTracker detector. (b) Cross section of a GigaTracker detector. The sensor (orange) is bump-bonded onto $2 \times 5$ TDCPix (blue) which are taped onto a cooling plate (grey). The cooling plate is then clamped on a PCB (green). (c) Schematics of a GigaTracker detector inserted into its vessel. (d) Picture of the backside (or cooling plate side) of a GigaTracker detector.

silicon plate with micro-channels of $70 \mu \mathrm{m} \times 200 \mu \mathrm{m}$ cross section etched in it is taped onto the ASIC side of the detector. A picture of such a cooling plate, produced by CEA Leti, is shown in Figure 6. The detectors is kept at $(-25 \pm 2){ }^{\circ} \mathrm{C}$ by circulating cold $\mathrm{C}_{6} \mathrm{~F}_{14}$ in the channels with a pressure of 3.5 bars for a flow of $3 \mathrm{~g} / \mathrm{s}$. In order to reduce the fluid pressure the channels are arranged in two cooling circuits as shown in Figure 6. The fluid is brought to channels with capillaries soldered on Kovar connectors themselves soldered onto the cooling plate as shown in Figure 4d.

\section{Performance}

The detector time resolution has been assessed during two beam times; one test beam in 2010 and the NA62 pilot run in 2014. In addition, tests were performed in the laboratory by injecting charge in the pixel sensor area with a laser pulse. The laser tests report a time resolution of $70 \mathrm{ps}$ RMS [4] for a charge injected at the pixel center equivalent to a MIP. Figure 7 shows the hit arrival time resolution as a function of the charge injected. 


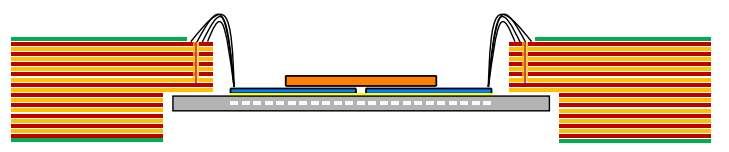

(a)

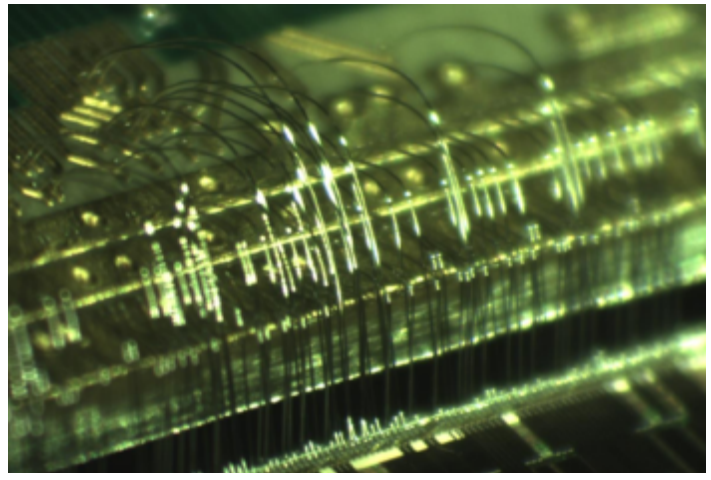

(b)

Figure 5: (a) Cross section of a GigaTracker detector. The sensor (orange) is bump-bonded onto $2 \times 5$ TDCPix (blue) which are taped onto a cooling plate (grey). The TDCPix are wire-bonded to a 14 layers PCB. (b) Picture of the wire-bonds.

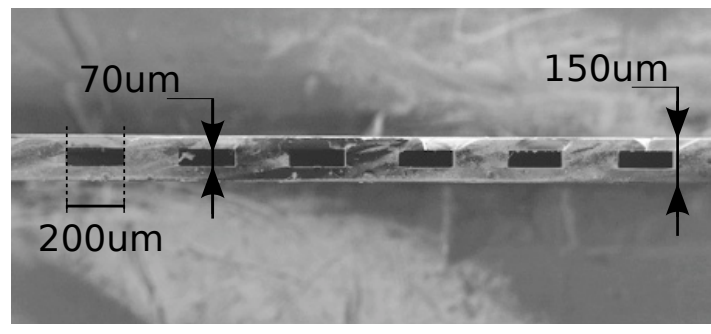

(a)

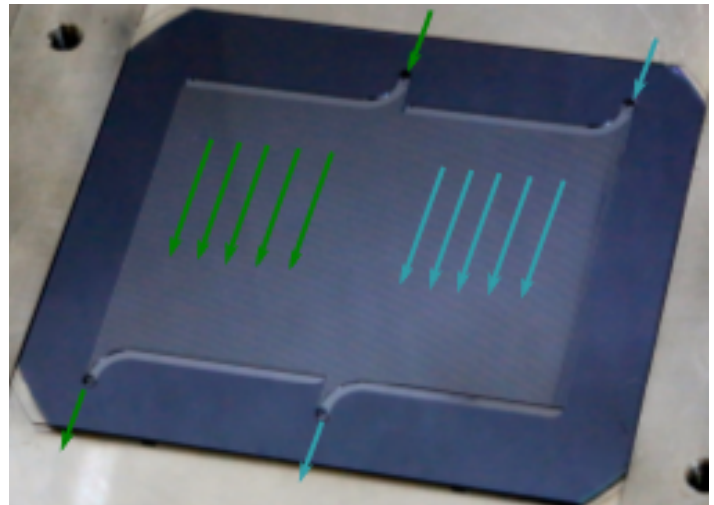

(b)

Figure 6: (a) Picture showing a cross section of the cooling plate. (b) Picture of a cooling plate closed up with glass, so that micro-channels are visible.

In 2010 a prototype of the detector was tested with a $10 \mathrm{GeV} / \mathrm{c} \pi^{+}$beam from the PS [5] which allowed to estimate the time resolution in conditions closer to the experiment ones. The hit arrival time resolution was measured to be 200ps as shown in Figure 8. Compared to the laser tests, the resolution is significantly larger.

This increase is explained by the contribution of two factors. First, during the beam test, particles were hitting the full pixel area while the laser was always shined at the pixel center. Since the electric field varies over the pixel area, the signal pulse shape varies too as shown in Figure 8. This pulse shape variation worsen the time resolution by 85 ps. Second, contrary to the laser case, the charge deposited by a particle along its path can change considerably, which results also in a pulse shape variation worsening the time resolution by 60ps.

Finally in 2014, two detectors were installed in the NA62 experiment and data could be col- 


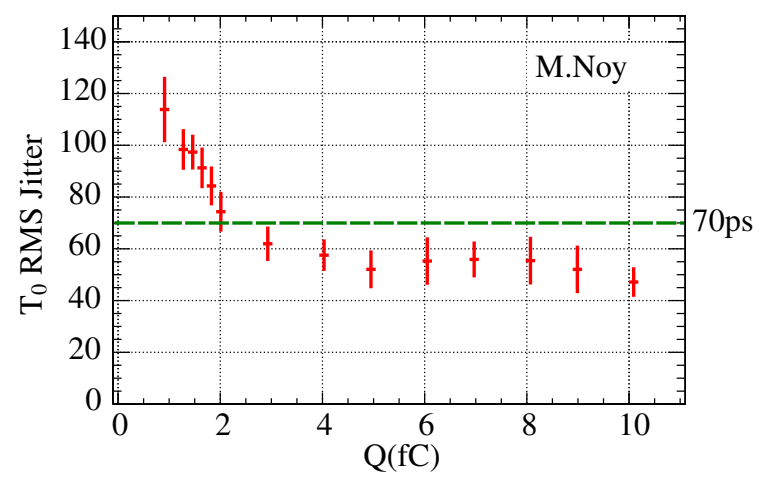

Figure 7: Hit arrival time resolution as a function of the charge injected from [4].

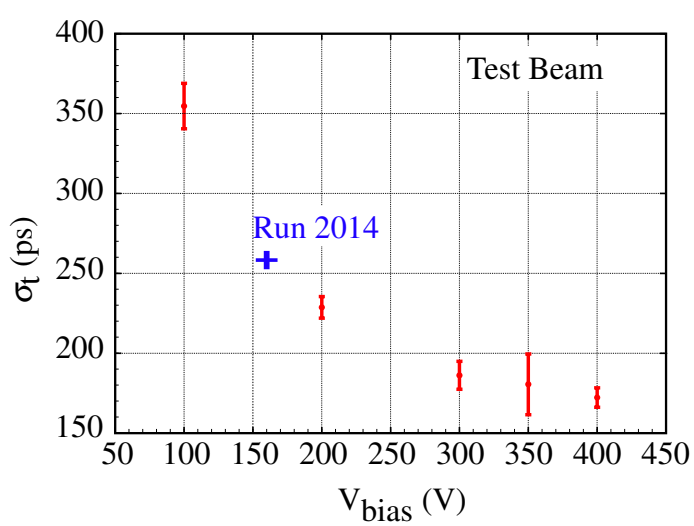

(a)

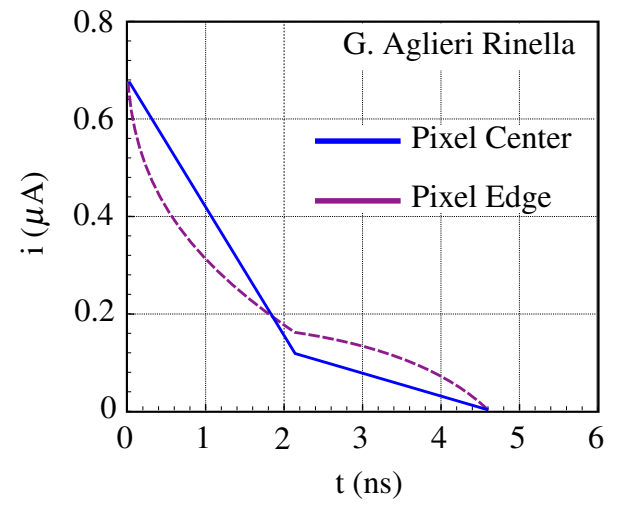

(b)

Figure 8: (a) Hit arrival time resolution as a function of the voltage bias applied to the sensor (modified from [5]. (b) Current sensor pulse as a function of time.

lected from one chip at the same location in both detectors ${ }^{2}$. All detectors were built with sensors at the nominal thickness. The detector in position 1 had a TDCPix thinned at $100 \mu \mathrm{m}$ and the one in position 3 at $450 \mu \mathrm{m}$. The cooling plates were $280 \mu \mathrm{m}$ thick. The hit maps observed in both stations are shown in Figure 9 and picture an excellent bump-bonding yield.

The detectors were operated with a bias voltage of $160 \mathrm{~V}$. In order to estimate the hit arrival time resolution, the time-walk correction has to be derived. This correction was obtained by correlating hits in station 3 with a fixed time-over-threshold with all hits in station 1 . The time-walk correction as a function of the time-over-threshold obtained with this method is shown in Figure 10. Using this correction a hit arrival time resolution of 260 ps can be achieved as shown in Figure 10. This value is in agreement with the ones obtained during the 2010 test beam for the same bias voltage as shown in Figure 8.

\footnotetext{
${ }^{2} \mathrm{~A}$ third detector was installed at the end of the run. It took data but with a chip position different from the one in the two other stations. As a result no hit collected with this station originated from the particle hitting the two other ones.
} 


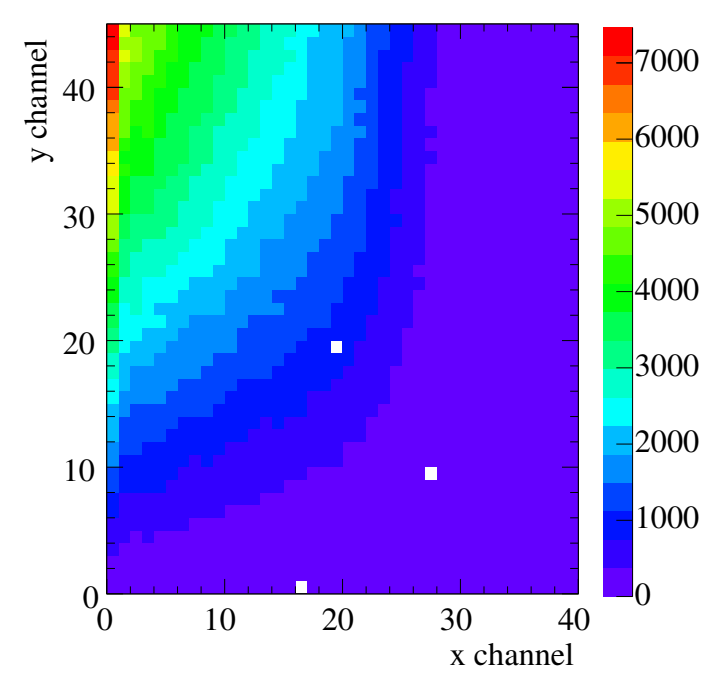

(a)

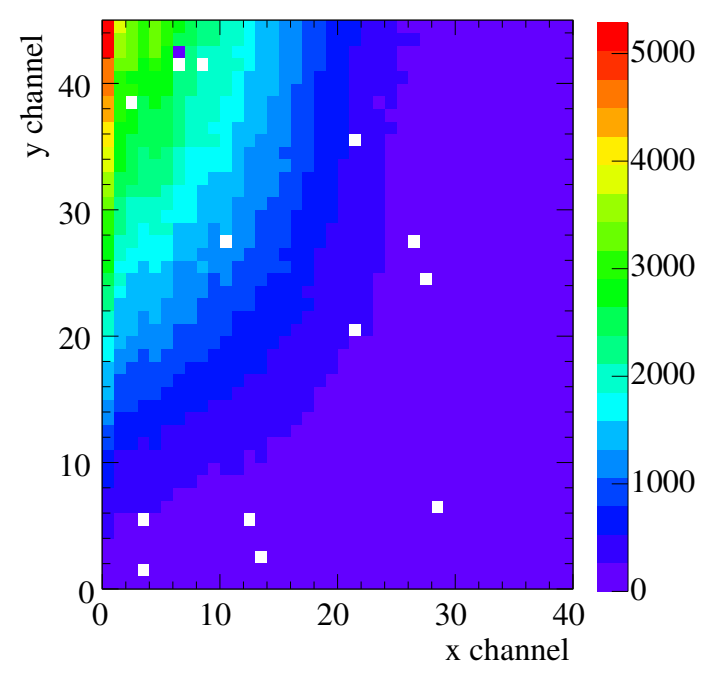

(b)

Figure 9: Hit maps in Station 1 (a) and Station 3 (b). Pixels at the left edge seems more illuminated as they are larger $(300 \mu \mathrm{m} \times 400 \mu \mathrm{m})$.

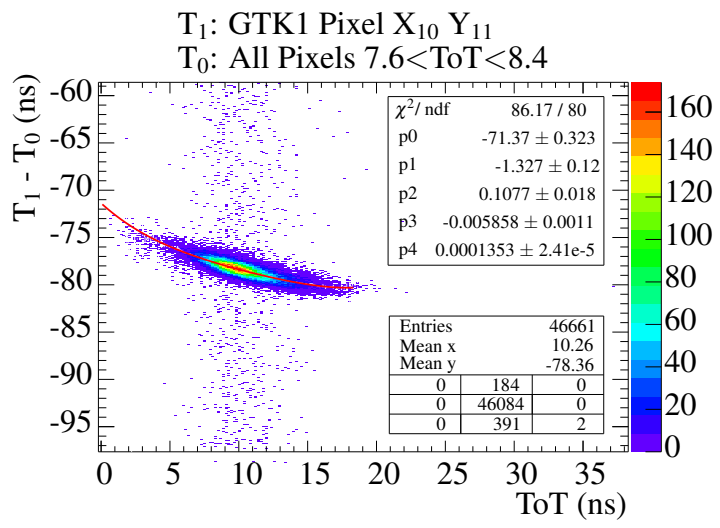

(a)

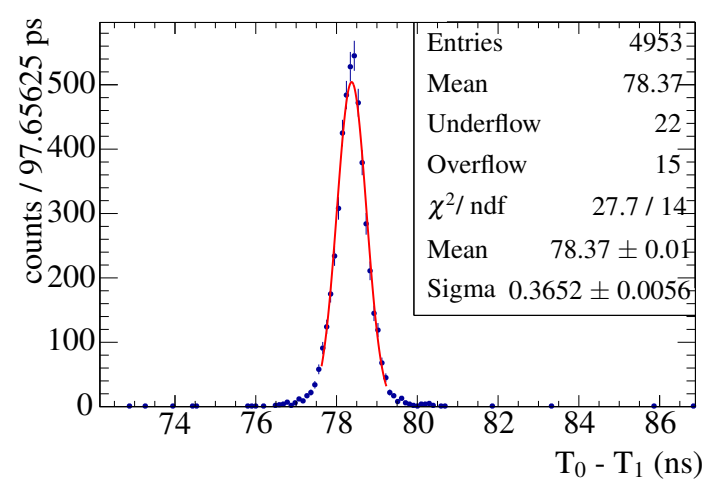

(b)

Figure 10: (a) Time-walk as a function of time-over-threshold. The data points are obtained from the 2014 run. The time-walk distribution mean as a function of the time-over-threshold is fitted with a polynomial function (red). (b) Time walk corrected time difference between hits deposed by the same particle in Station 3 and Station 1. The data points are obtained from the 2014 run and are fitted with a Gaussian function (red). The hit arrival time resolution can be derived from this distribution width dividing it $\sqrt{2}$ (i.e. 260ps). This is possible with the assumption that the time measurements are uncorrelated and have individually the same time resolution.

\section{Conclusions and Prospects}

The GigaTracker is an ambitious project aiming at measuring the momentum and arrival time of particles in a beam with a flux as high as $1.3 \mathrm{MHz} / \mathrm{mm}^{2}$. A dedicated ASIC, the TDCPix, has been designed for this purpose. Tests and first operations in the experiment demonstrate that a resolution as good as 200ps can be achieved. Moreover, the detector implements for the first time in high energy physics a micro-channel cooling which allow to keep the material budget to less 
than $0.5 X_{0}$ per station.

The major challenges of the detector construction have been solved and detectors close to the nominal design have been produced. The next detector production will try to bridge the gap and match these specifications. As far as performance is concerned, the first detectors have achieved the designed time resolution. The next NA62 run will allow to study more in details the momentum and angular resolutions, the efficiency of the detector at rates close to the nominal one and, the effect of radiation induced sensor aging.

\section{References}

[1] Brod, Gorbahn, Stamou Phys. Rev. D 83, 34030 (2011)

[2] Ceccucci et al., CERN-SPSC-2005-013

[3] G. Aglieri Rinella et al., Physics Procedia 37, (2012)

[4] M. Noy et al., J. Instrum. 6, C01086 (2011)

[5] M. Fiorini, Nucl. Instrum. A 718, (2013) 